\title{
Science for Sustainability: Using Societal Metabolism Analysis to check the robustness of European Union policy narratives in the water, energy and food nexus
}

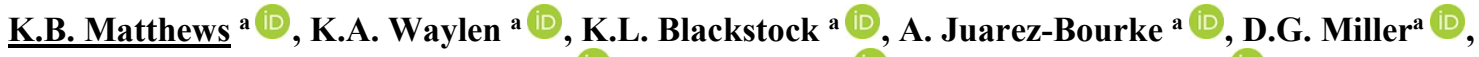 \\ D.H. Wardell-Johnson a ${ }^{(D)}$, M. Rivington ${ }^{a}$ (iD) and M. Giampietro ${ }^{\text {b, }}$ (iD) \\ ${ }^{a}$ The James Hutton Institute, Aberdeen, Scotland \\ ${ }^{b}$ Institut de Ciència i Tecnologia Ambientals, Universitat Autonoma de Barcelona, Catalunya \\ 'Institució Catalana de Recerca i Estudis Avançats (ICREA), Barcelona, Catalunya
}

\section{Email: keith.matthews@hutton.ac.uk}

\begin{abstract}
This paper is an output of an ongoing EU Horizon 2020 project (MAGIC) that aims to better understand how EU water, food, energy, waste and biodiversity policies link with each other and with EU climate and sustainability goals, framed in terms of the nexus concept. The project conducts transdisciplinary research with policy makers using an approach termed Quantitative Story Telling (QST), as an interface between science and policy domains. QST combines semantic (qualitative) and formal (quantitative) approaches to assess the plausibility, normative fairness and analytical coherence of narratives being used by stakeholders to justify either the status quo or alternative policy positions for the EU. The paper focuses on those aspects of the MAGIC analysis highlighted by external reviewers of the project as being most insightful and having the most potential value to a wider community of practice concerned with supporting or evaluating sustainability related policies. The paper outlines the process of QST used and the quantitative method used, multi-scale societal metabolism analyses (SMA) assessing the funds of land and human time needed to create the flows of materials, energy and money that reproduce and maintain the identity of the system of interest.
\end{abstract}

As one of the five MAGIC policy studies, the authors focused on a key EU Common Agricultural Policy (CAP) narrative. CAP is a policy which is now expected to deliver multiple objectives across policy domains, but as implemented, potentially contributes to a tension between supporting competitiveness and delivering public goods. High-level findings that quantify aspects of this tension are presented, followed by specific technical issues found when conducting the analysis. The paper then reflects on the authors' use of these data to discuss with policy-makers issues where the tension between competitiveness and public goods are most stark; a more interpretive, qualitative phase of analysis that builds on the quantitative analysis.

The outputs of the analysis used within the CAP QST imply the need for policy makers to consider alternative issue framings, otherwise they risk appearing to make only a rhetorical commitment to defining and delivering EU sustainability goals. The societal metabolic framing used in MAGIC highlights the biophysical underpinnings of EU farming systems; their dependence on non-renewable resources and the pressures generated by them that degrade ecosystem functions or services. A societal metabolic framing also means considering multiple scales, since otherwise EU policy is blind to the effects it has on sustainability beyond the borders of the EU.

If research impact is defined in terms of acknowledged change in stakeholders' concepts or behaviours (an expected impact for the project by funders) then to date, there has been limited 'success'. While the rhetoric of 'evidence-based policy' remains prominent, it remains extremely challenging to engage with policy makers in deliberation on evidence that challenges conventional narratives. This was the case even for staff with extensive experience of inter- and transdisciplinary working at the science-policy interface.

In conclusion, science for sustainability policy could benefit from adopting the approaches like QST, which can integrate and balance the semantic and formal parts of science for policy research. For the wider sciencepolicy community of practice, the key insight is that for processes like QST the key decisions are made at the interfaces between the sematic and formal phases of analysis (what is modelled and why) and the formal and semantic phases of analysis (what the outputs mean and why they shouldn't be ignored).

Keywords: Societal metabolism, sustainability, nexus, science-policy, common agricultural policy 


\section{INTRODUCTION}

This paper is an output of an EU Horizon 2020 project (MAGIC) being conducted between 2016-2020 to better understand how EU water, food, energy, waste and biodiversity policies are linked together and to EU climate and sustainability goals, framed in terms of the Nexus concept (Cairns and Krzywoszynska, 2016). The intention within MAGIC is to conduct transdisciplinary research with policy makers using an approach termed Quantitative Story Telling (QST) as an interface between science and policy domains. QST combines qualitative and quantitative analysis methods to assess the plausibility, normative fairness and analytical coherence of narratives being used by stakeholders to justify either the status quo or alternative policy positions for the EU. Narrative is used here (semi-colloquially) to indicate public statements made by decision makers, particularly those that define objectives, the means by which they are to be achieved and the evidence / methods of analysis that are considered in decision making (i.e. the epistemic box that frames the analysis and policy making). The process of QST draws philosophically on post-normal science for governance. Here the quality of analytical outcomes depends on clarifying, in a dialogue between policy makers and researchers, the choices that have shaped the content of the 'evidence base' and the modes of analysis considered salient and credible. The QST is not therefore concerned with refinement of the minutiae of evidence but rather questioning whether existing science-policy consensuses for major policy questions such as sustainability are ignoring existential threats because they take too narrow a view of the challenges faced by the EU.

The EU Common Agricultural Policy (CAP) remains the largest financial instrument through which the management of European terrestrial natural resources can be influenced. While the CAP is not a water, energy or food policy per se it wields immense influence via the financial support provided to, and the regulation of, agricultural systems. This makes the CAP the central policy in shaping the Nexus in Europe and thus an appropriate lens though which to assess how policy integration or coherence can be better delivered.

The narrative chosen for analysis with policy stakeholders in MAGIC was that "CAP aims to ensure European agricultural competitiveness in the world market and aims to deliver public goods such as biodiversity conservation, water quality and climate change mitigation. These aims are in opposition". The CAP team's analysis of the competitiveness and public goods narrative aimed for a pan European coverage and taking a small number of non-equivalent perspectives on EU farming systems, these characterised imports and exports of agricultural commodities, the role of subsidy in maintaining financial viability, the intensities of inputs use for key sectors and the aggregate impact of EU production systems.

This paper draws from those aspects of the MAGIC analysis highlighted by external reviewers of the project as being most insightful and having the most potential value to a wider community of practice concerned with supporting or evaluating sustainability related policies. The paper focuses on the higher-level learning points that relate to process of conducting research at the science policy interface rather than the details of the analysis; available in Matthews et al. (2018). Smaller numbers of policy and EU specific insights are also included where the authors think that they are likely to be relevant to a wider international community of practice.

\section{METHODS}

QST is set out in more detail in a previous MODSIM paper (Matthews et al., 2017) and is summarised in Figure 1 , with phases of semantic (qualitative focused) and formal (quantitative focused) analysis. The process of formal quantitative analysis is thus profoundly shaped by the prior semantic analysis, but how the quantifications are understood and their implications for policy also subject to interpretation and debate with stakeholders.

Societal metabolism accounting (SMA) methods such as MuSIASEM (Giampietro et al., 2014) are the integrative and analytical core of MAGIC. SMA focuses analysis on the funds of land and human time needed to create the flows of materials, energy and money that reproduce and maintain the identity of the system of interest (e.g. current patterns and trajectories of consumption). The analysis considers both extents of resource use and intensities since

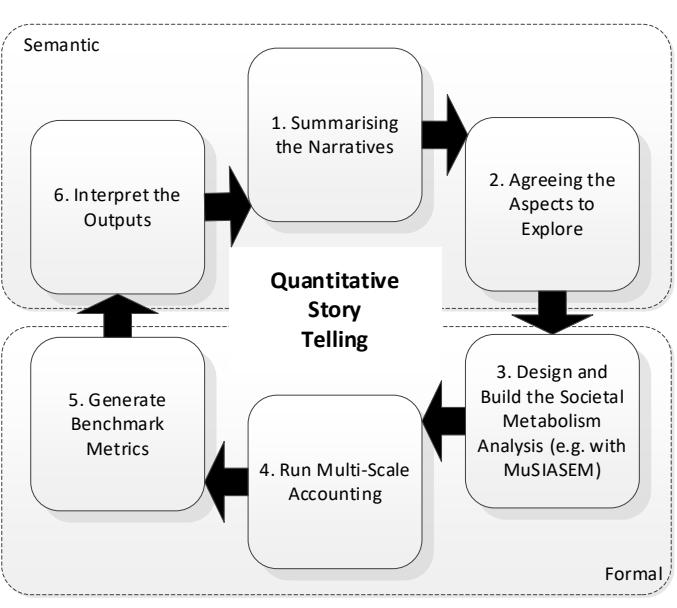

Figure 1. The process of Quantitative Story Telling each provides useful perspectives. For example, increase in efficiency per hectare of water use for a land use may be entirely offset by land use change that means the mix of land uses present now has a greater proportion 
of higher demand crops - e.g. more irrigated vegetables. The analysis is conducted simultaneously across scales (geographical or sectors) to highlight key externalisation effects and dependencies that may undermine long-term security. SMA uses reformulations of existing statistical datasets and coefficients derived from literature or statistical analyses.

The analytical coherence of the policy narratives is assessed using FVD benchmarks, that is their feasibility (i.e. their compatibility with the limits of the biosphere that are beyond human control)), their viability (i.e. their dependence on the existing socio-technical systems under human control such as the availability of: trade partners; low cost agricultural commodities and energy carriers; or financial subsidy) and their desirability (reflecting distributional and acceptability issues).

\section{SUMMARY OF FINDINGS}

This section provides a very high-level summary of the findings from the MAGIC CAP analysis (again see Matthews et al. (2018) for more detail). This is included to provide enough context for the more detailed discussion of the insights gained from applying QST, anticipated to be of greater interest to the MODSIM science-for-policy and applied modelling communities of practice.

Analysis of international trade in agricultural products highlighted the dependence of EU agriculture on external resources, e.g. feedstocks. These increase the competitiveness of EU agriculture, but their production can undermine public good provision beyond the borders of the EU by for example encouraging conversion from woodland to cropland. The analyses of productivity across agricultural sectors, illustrated the role of subsidies in keeping farming viable, but noted significant windfalls for sectors, presently viable without subsidy. Despite some opportunities for increases in efficiency of resource use, the magnitude of outputs from farming systems are still largely driven by the level of inputs. Any significant reduction in intensity of input use to improve the provision of public goods would seem to require an acceptance of less output. This suggests that if CAP is intended to achieve a more sustainable balance of competitiveness and provision of public goods, then it may need to support measures to reduce the demands being placed on the farmed areas of the EU.

The technical issues identified by the analysis were that it is challenging to link the pressures generated by an individual production system to their environmental impacts since the pressures to impacts relationships are mediated by the vulnerability of the environment in which they occur and the mix of other production systems present. However, at an aggregate level it is possible to illustrate the size and shape of the pressures being generated i.e. the relationships between input (e.g. $\mathrm{N}$ ), outputs (embodied $\mathrm{N}$ in yields) and emissions, looking at extents, intensities and emissions. The focus on $\mathrm{N}$ inputs, outputs and emissions extents helped illustrate the importance of such metrics, as opposed to the more usual intensity metrics (e.g. per unit of area). |The cumulative effect of lower intensity but large extent emissions can undermine delivery of EU GHG emissions commitments. The soil erosion analysis seemed to suggest a more fundamental challenge, in that in nearly all member states soil erosion in crop-based production systems was exceeding the rate of soil renewal processes.

The spatial representation of analysis outputs was important in reinforcing the heterogeneity of production systems across the EU. Whilst the function of some production systems is the same (for example specialist cereal production), the size of farms, their mix of activities and input intensity vary considerably. Combined with the mix of other production systems present, their impacts on the biosphere is also highly variable. There are data on state-of-the-environment variables produced with a fine granularity, but these are not easily linked to production systems data such as those within the EU Farm Accountancy Data Network. FADN focuses on the financial performance of farms but also increasingly includes biophysical parameters such as yield and quantifies some agricultural inputs and outputs in physical terms. The analysis here has illustrated options for future analysis to supplement FADN data with research derived datasets, and the potential value of including additional physical and management data within the FADN data in future.

The research team interpreted the QST analyses as supporting the narrative that the pursuit of competitiveness and delivery of public goods were in tension: however, stakeholders who participated in a 'closing the QST loop' workshop were not necessarily convinced by our findings. This reluctance is not surprising given the unconventional nature of the QST and SMA that challenges traditional approaches to CAP evaluation and its presentation at the time when a consensus has been carefully fashioned within the EU institutions on the new CAP objectives. These difficulties were compounded by those encountered by a research team doing a societal metabolism-based analysis on CAP policy for the first time and the fact that the researchers are still building trust and relationships with stakeholders. Taken altogether, these experiences suggest that using QST to openup new conversations is challenging but not impossible. 


\section{REFLECTIONS ON USING QUANTITATIVE STORY TELLING}

This section reflects in more detail on what has been learnt from this cycle of QST in terms of potential future analyses of CAP and the Nexus.

\subsection{Insights for policy}

While the stated objectives for CAP are now mapped onto the UN Sustainable Development Goals (SDGs) (European Commission, 2019) and the policy is presented as a flagship example for delivering policy integration and coherence across sectors (European Association of Agrcultural Economists, 2019) it is still challenging to demonstrate how mechanistically the CAP drives progress towards the EU sustainability goals. Further, if commitments to the Paris Climate Accords or more ambitious zero emissions ambitions are to be met, then CAP and other policies may need to support a more fundamental realignment of EU agriculture accepting that the current extent of outputs may not be sustainable in the long term.

1 - EU agriculture depends on imports and by doing so it reduces its apparent environmental footprint. How the CAP shapes EU production systems has implications far beyond the borders of the EU. Imports (such as livestock feeds) means drawing on resources and causing environmental impacts beyond the EU. Since 2006 exports (e.g. to north Africa) also have a role in providing food security beyond the EU. A more comprehensive analysis of how CAP shapes demand from beyond the EU and the balance sheet of benefits and burdens in social metabolic terms would be informative in saying how CAP and EU agriculture contribute to the SDG's. Consideration of how such externalised impacts are accounted for within CAP impact assessment and related policymaking may also be needed (e.g. better integrating both supply and demand side accounting).

2 - Irrigated crops generate high value outputs but depend on fossil resources. Irrigated agricultural systems are extensive and are intensive users of water. They are important in terms of their financial value as well as the source of key elements of the EU diet. Yet in many regions such systems face challenges from the depletion of fossil water resources exacerbated by climate change. The potential role of the CAP in supporting systems in their adaptation is an interesting Nexus issue to follow up.

3 - Loss of soil is a fundamental challenge. Given the evident draw down on the fund of soil by water and wind erosion, the question becomes one of how EU production systems would have to change to be compatible with soil forming rates. Can production be changed such that erosion is reduced (e.g. through min-tillage systems or cover crops) and if so, what are the implications for the balance of cost and benefits for the biosphere, the supply system and the consumer. Alternatively, are the rates so high that there needs to be a fundamental change in the level of output that can be expected from these systems with implications for EU food security and/or a reduction in the capacity of the EU to generate exports. The former is certainly more palatable in policy terms as it is compatible with a narrative of efficiency, productivity and competitiveness but is it realistically achievable?

\subsection{Insights for Nexus analysis}

The policy insights highlight the continuing need for research that identifies where current framings are inadequate but also suggests the need for analyses in which new framings and evidence can be evaluated and used to shape policy and practice.

1. Move from WEF to WEFE Nexus. Wiegleb and Bruns (2018) observe that much of the Nexus scholarship tends to focus on the built environment and engineering solutions (i.e. the technosphere). They call for more attention to the environmental dimensions (moving from WEF to WEFE); and for Nexus analysts to take a spatially explicit approach. The societal metabolism approach in MAGIC links bio- and technosphere and explores how to map these interactions in physical space as well as across time series. However, our analysis illustrates how difficult spatial analysis can be when constrained by data availability at the appropriate resolution; and since spatial data relationships are complex to visualise in easily interpretable ways.

2. Consider food security at multiple scales. The societal metabolism analysis highlighted the degree to which EU agriculture depends on external sources for its food security. It also highlighted that any consideration of the sustainability of EU agriculture should also consider its footprint beyond the EU otherwise there is a danger that sustainability objectives are achieved locally by externalising impact (e.g. GHG emissions). Quantifying the intra-EU flows of materials within and between EU sectors and member states (and if possible, regions) in physical terms would allow for an analysis of the role of EU policy delivering food security within the EU. It would though also potentially highlight where intra-EU trade is driving processes of specialisation that are potentially environmentally harmful. 
3. Provision of public goods - here the challenge is trying to maintain breadth of scope while retaining meaningful depth. The temptation is always to take a narrower perspective (where more data is available) but the danger here is of potentially studying the wrong part of the system. A priority would be to look further at changes in extents and intensity in space for production systems (crop and livestock) to characterise areas experiencing abandonment and areas where greater surpluses are being generated (some of which are contributing to exports).

4. Better representing energy in the analysis. The analysis presented did not tackle the energy use and embodied energy aspects of supply and production systems. This could to a degree be rectified with bottom up analyses. Such analyses would be immeasurably strengthened however if the FADN dataset could provide a top-down characterisation of energy use in physical terms (both amounts and types of energy carriers used) and the power capacity of plant and machinery deployed.

5. Reconceptualising competitiveness? Increased competitiveness is a key objective of CAP, but in terms of desirability, questions were raised by MAGIC reviewers. These included, who benefits from increased competitiveness, differentiating between the outcomes for primary producers, processors, retail and consumers. This would potentially provide insights across a range of nexus policies where trade-offs may need to be considered - e.g. food prices, rural employment, the roles of agribusiness and smaller producers (all aspects listed in the new CAP objectives). There may also be value in trying to assess the role of the CAP in promoting adaptability in EU farming systems - that is the ability to remain competitive in the face of change and/or uncertainty over longer time scales. Social-ecological systems theory would argue that greater adaptability may entail farming not being optimized to current circumstances and thus having a degree of competitive disadvantage in the short term (Holling et al., 2002).

\subsection{Insights for conducting QST}

This section covers what has been learnt about QST as a process and how this could influence future applications. These lessons are presented for consideration by other researchers wishing to adopt a QST type methodology.

1. Choice of narrative and the mode of enquiry. It is important to ensure that those engaging with the interpretation phases of QST outputs agree that the quantified results from the societal metabolism analysis are salient. Given that the individuals involved in 'closing the QST loop' may not be the same as those involved in choosing the narrative at the start, it might be useful to regularly rehearse the narrative, to reflect over time on how it is being received by an ever-shifting and widening group of stakeholders. In our case, given the degree of debate that accompanied the selection of our narrative, on the tension between competitiveness and the environment, it may have been wise to reword it before presenting the QST outputs at the end of the loop Its provocative framing exacerbated discomfort with our presentation, and may have spurred debate of, and resistance to, the quantified evidence. However, it is hard to continually open up the choice of narratives and still complete a quantification of a bounded problem. One solution might be to quantitatively analyse more than one narrative; but this of course implies more effort and resources and is therefore easier to do once researchers gain more confidence/competence in the application of a societal metabolism approach.

The application of QST to CAP is in 'diagnostic mode' i.e. analyses that (re-)present information about current systems, whereas the more readily accepted QST applications, within MAGIC to date, have been in 'simulation mode' (exploring or imagining alternative futures). Potentially these different modes of application have very different challenges and it might be useful to reflect on what can be learnt about working diagnostically. Perhaps it is easier or more comfortable for stakeholders to engage with imagined futures rather than analyses that may appear to imply criticisms of their recent and ongoing activities.

2. Building credibility. Working with data sets and within existing procedures familiar to stakeholders is important - it builds credibility and illustrates how something as radical as societal metabolism and QST could support their institutional processes. Likewise, it is important to illustrate how the analysis might fit with the policy cycle. In some ways, the timing of the MAGIC analysis was poor, as the CAP in 2018-19 is going through a process of closing-down policy options and focussing on implementing agreed objectives. This may help explain why our participants were encouraging us to focus on CAP as part of the delivery of wider goals e.g. the EU commitments to the UN Sustainable Development Goals (SDGs) which is a policy domain in a more open phase of deliberation.

3. Complexity of SMA and the SMA outputs. There is a significant challenge in harnessing the power of a societal-metabolism analysis. The richness and multi-dimensional nature of the societal-metabolism analysis means it is inherently less easy to summarise than other methodologies that collapse complexity into a single 
metric or single unit of measure. This is especially true for a phase of QST analysis where narratives are being opened-up and scrutinised from multiple perspectives (an inherent part of any Nexus study). While it is easy in scientific terms to take a falsification approach, showing individual aspects when narratives are not robust, this can be seen as unhelpfully critical, if alternative coherent narratives linking objectives and the means of achieving them are not offered (solutions). Marshalling the outputs from societal-metabolism analysis in ways that can be influential within policy processes therefore remains challenging. The technical aspects of using societal-metabolism based analyses could perhaps be enhanced by having more interactive software tools that can be used by researchers but with stakeholders. Yet this presupposes that it is possible to make time in stakeholders' busy schedules in which such interactions can take place. This is an institutional challenge for policy stakeholders, how to generate the "space" for their staff to make a potentially substantial effort to add new methods such as societal-metabolism to their lexicon. The answer to the institutional aspect is perhaps that such capacity can only be justified within an active policy-making processes. Therefore, including experimental analyses to complement established policy support methods should perhaps be considered.

4 The challenge of operationalising FVD. The ideas in FVD proved very difficult to apply within the specific and time-limited policy engagement process of MAGIC. It was the absence of a clear understanding of how to operationalise the concepts of FVD that challenged our application, rather than lacking data on which to base benchmarks. Thus, the authors suggest that for QST it is important to discuss the framing of the FVD benchmarking early and agree how this will shape the content and conduct of the preceding stages of the societal metabolism analysis. There is thus a potentially complex coevolution of the sematic and formal components of the analysis that can change over time as insights are gained (breaking the neat circularity and sequential ordering implied by the numbering of stages in Figure 1). Also worth noting is that while the societal metabolism analyses being used are very flexible, any formalism will serve to limit the scope of what can be considered in an analysis of policy narratives, so care needs to be taken that as far as possible the issues shape the analysis or at least the shaping of the issues by the formalism is done consciously and explicitly.

Reflecting on the benchmarks, Feasibility is the most clear-cut conceptually but acknowledging biophysical thresholds and limits remains highly contested. Viability is problematic given its multi-dimensional nature (i.e. technical, economic, institutional or by externalisation). Also, if a policy is implemented, it is selfevidently viable at least in the short term. What viability is contingent on may be the key insight gained from SMA, e.g. trade or fossil resources. Finally, Desirability is currently the least well-defined benchmark, particularly when working in diagnostic mode. For implemented policies, some decision on desirability was made, and reopening the deliberation on this kind of political decision is rarely welcome. For future policies (via analysis in simulation mode) desirability benchmarking has more potential. It is perhaps though in desirability assessment where the 'epistemic box' for policy is least open. This emphasises the need for researchers to engage actively with multiple-stakeholders during the semantic interpretation 'closing the loop' phase of QST, as the outputs cannot be expected to speak for themselves.

5. Norms for science-policy engagement and the need for multiple QST cycles. Our experience has given us useful insights into norms of interactions with researchers within DG Agri. In particular, it is notable that individuals in the DG tend not to engage directly with scientists to shape analysis and so were unaccustomed to being interviewed or discussing research-in-progress. Likewise, our team were new to the 'norms' of science-policy interfaces within the European Commission. Therefore, we were all beginners and our success or failure in implementing QST should be understood in that context. Our experience also indicates challenges in implementing some of the recommendations often made by the literature on science-policy interfaces. For example, repeated interactions and open conversations are often recommended (e.g. Young et al., 2014) but engaging at an early stage can carry risks that a preliminary analysis is not seen as credible or the team does not feel fully confident and competent.

It may also be important to distinguish what can be achieved from different cycles of QST. The authors experiences suggest that using QST to open-up new conversations will take time, with different cycles of engagement achieving different degrees and types of influence. QST is explicitly designed to be a cycle that can be repeated over time; doing so is likely to build familiarity with the method, outputs and between individuals (subject to the limitations from inevitable staff changes and turnover).

\section{CONCLUSIONS}

This phase of MAGIC analysis was intended to 'learn through doing' both the processes of transdisciplinary working (conducting the QST process with stakeholders) and mastering the theory and implementation of societal-metabolism based analyses. The intent was that, before trying to engage in the more complex issues of multi-policy integration in the WEF nexus, it would be valuable to have engaged with key individual policy domains and to have built a degree of credibility. This process has also allowed researchers the opportunity to 
become more familiar with institutional structures and processes so that opportunities for applying the methods to real policy issues could be identified and, in some cases, exploited to increase the impact of the research. The degree of success in this regard varied between the MAGIC research teams within MAGIC (see the reports in the MAGIC document repository). Those with greater experience of societal metabolism analysis progressed well the formal aspects and, in some cases, had substantial buy-in from stakeholders. The key caveat to this conclusion is that success can depend significantly on how well the messages from the research align with the existing perspectives of the stakeholders. For example, a critique of the performance of the CAP tends to be welcomed by stakeholders with an environmental perspective much more than those from agriculture. In neither case is it easy for research-based outputs to be influential in terms of engendering conceptual change.

The paper has provided insights from a research project that has been conducted by staff with extensive experience of inter- and transdisciplinary working at the science-policy interface. Despite their experience the degree of success was limited. While the rhetoric of 'evidence-based policy' remains prominent it is extremely challenging to engage with policy makers when the outcome being sought is conceptual change, i.e. the reframing of the evidence included within a policy making process. The need for reframing is apparent to the authors since otherwise in many cases there is a danger of policy being blinkered by established processes of analysis (theories, methods and what is considered evidence). The process of QST has the potential to better integrate and balance the semantic and formal parts of science for policy research, to help enact processes of progressive policy change that can recognise the existential sustainability challenges. The main conclusion of the paper for the wider science-policy community of practice is that for processes like QST the key decisions are made at the interfaces between the sematic and formal (what is modelled and why) and the formal and semantic (what the outputs mean and why they shouldn't be ignored).

\section{ACKNOWLEDGMENTS}

This project is funded by the European Union's Horizon 2020 Research and Innovation Programme under grant agreement No. 689669. The present work reflects only the authors' view and the funding Agency cannot be held responsible for any use that may be made of the information it contains.

\section{REFERENCES}

Cairns, R., Krzywoszynska, A., 2016. Anatomy of a buzzword: The emergence of the water-energy-food nexus' in UK natural resource debates. Environmental Science \& Policy 64, 164-170.

European Association of Agrcultural Economists, 2019. Agricultural policy for the environment or environmental policy for agriculture? , 172nd Seminar. EAAE, Brussels.

European Commission, 2019. Mappng EU Policies into SDGs, http://knowsdgs.jrc.ec.europa.eu/mappingpolicies

Giampietro, M., Aspinall, R.J., Ramos-Martin, J., Bukkens, S., 2014. Resource Accounting for Sustainability Assessment: The Nexus between Energy, Food, Water and Land use. Routledge, 250 pp.

Holling, C.S., Gunderson, L.H., Peterson, G., 2002. Sustainability and Panarchies., in: Gunderson, L.H., Holling, C.S. (Eds.), Panarchy: Understanding transformations in human and natural systems. Island Press, Washington, pp. 63-102.

Matthews, K.B., Blackstock, K.L., Rivington, M., Waylen, K.A., Miller, D.G., Wardell-Johnson, D., Kovacic, Z., Renner, A., Ripa, M., Giampietro, M., 2017. Delivering more than the "Sum of the Parts": using Quantitative Storytelling to address the challenges of conducting science for policy in the EU land, water and energy nexus. , in: Syme, G., Hatton MacDonald, D., F., B., Piantadosi, J. (Eds.), MODSIM2017, 22nd International Congress on Modelling and Simulation. Modelling and Simulation Society of Australia and New Zealand, p. 7.

Matthews, K.B., Blackstock, K.L., Waylen, K.A., Juarez-Bourke, A., Miller, D.G., Wardell-Johnson, D.H., Rivington, M., 2018. Report on the Quality Check of the Robustness of the Narrative behind the Common Agricultural Policy (CAP). MAGIC (H2020-GA 689669) Project Deliverable 5.5, p. 65.

Wiegleb, V., Bruns, A., 2018. What is driving the Water-Energy-Food Nexus? Discourses, knowledge and politics of an emerging resource governance concept. Frontiers in Environmental Science 6, 128.

Young, J.C., Waylen, K.A., Sarkki, S., Albon, S., Bainbridge, I., Balian, E., Davidson, J., Edwards, D., Fairley, R., Margerison, C., McCracken, D., Owen, R., Quine, C.P., Stewart-Roper, C., Thompson, D., Tinch, R., Van den Hove, S., Watt, A., 2014. Improving the science-policy dialogue to meet the challenges of biodiversity conservation: having conversations rather than talking at one-another. Biodiversity and Conservation 23, 387-404. 